\title{
E-DEEC- Enhanced Distributed Energy Efficient Clustering Scheme for heterogeneous WSN
}

\author{
Parul Saini \\ Department of Computer Science \& \\ Engineering, \\ Dr. B. R. Ambedkar National Institute of \\ Technology, \\ Jalandhar ,Punjab, 144011, India \\ psgs1012@gmail.com
}

\author{
Ajay.K.Sharma \\ Department of Computer Science \& \\ Engineering, \\ Dr. B. R. Ambedkar National Institute of \\ Technology, \\ Jalandhar ,Punjab, 144011, India \\ sharmaajayk@nitj.ac.in
}

\begin{abstract}
Many routing protocols on clustering structure have been proposed in recent years. In recent advances, achieving the energy efficiency, lifetime, deployment of nodes, fault tolerance, latency, in short high reliability and robustness have become the main research goals of wireless sensor network. Many routing protocols on clustering structure have been proposed in recent years based on heterogeneity. We propose EDEEC for three types of nodes in prolonging the lifetime and stability of the network. Hence, it increases the heterogeneity and energy level of the network. Simulation results show that EDEEC performs better than SEP with more stability and effective messages.
\end{abstract}

\section{Introduction}

Wireless sensor networks is the network consisting of hundreds of compact and tiny sensor nodes which senses the physical environment in terms of temperature, humidity, light, sound, vibration, etc. These sensor nodes gather the data from the sensing field and send this information to the end user. These sensor nodes can be deployed on many applications. Current wireless sensor network is working on the problems of low-power communication, sensing, energy storage, and computation.

Hierarchical-based routing is a cluster based routing in which high energy nodes are randomly selected for processing and sending data while low energy nodes are used for sensing and send information to the cluster heads. Clustering technique enables the sensor network to work more efficiently. It increases the energy consumption of the sensor network and hence the lifetime [1].
Clustering can be done in two types of networks, homogeneous and heterogeneous networks on the basis of energy. Homogeneous are those in which nodes have same initial energy while heterogeneous networks are those in which nodes have different initial energy. Many Clustering algorithms have been proposed for homogeneous wireless sensor networks such as LEACH [2], PEGASIS [3], and HEED [4] which does not perform well in heterogeneous networks. SEP [5] uses two types of nodes normal and advanced nodes. Advanced nodes have more energy than normal ones. It prolongs the stability period of the network. It also does not fit for networks having more than two types of energy.

DEEC [6] is clustering-based algorithm in which cluster head is selected on the basis of probability of ratio of residual energy and average energy of the network. In this algorithm, node having more energy has more chances to be a cluster head. It prolongs the lifetime of the network. Ours E-DEEC follows the thoughts of DEEC and adds another type of node called super nodes to increase the heterogeneity.

The rest of the paper is organized as follows: Section 2 contains the related work done. Section3 explains the radio energy dissipation model, Section 4 and 5 gives the network model and assumption used followed by section 6 which describes the cluster head selection method. Section 7 lists the performance metrics used for the simulation which gives the results shown in section 8 .

\section{Related Work}

For homogeneous wireless sensor networks Heinzelman, et. al. [2] introduced a hierarchical clustering algorithm for sensor networks, called Low 
Energy Adaptive Clustering Hierarchy (LEACH). LEACH is a cluster-based protocol, which includes distributed cluster formation. LEACH randomly selects a few sensor nodes as cluster heads $(\mathrm{CHs})$ and rotates this role to evenly distribute the energy load among the sensors in the network [1]. PEGASIS [3] is a chain based protocol which avoids cluster formation and uses only one node in a chain to transmit to the BS instead of using multiple nodes.

Manjeshwar et. al. proposed Threshold sensitive Energy Efficient sensor Network protocol (TEEN) [7]. TEEN pursues a hierarchical approach along with the use of a data-centric mechanism. the cluster head broadcasts two thresholds to the nodes. These thresholds are hard and soft thresholds for sensed attributes. TEEN is not good for applications where periodic reports are needed since the user may not get any data at all if the thresholds are not reached. Manjeshwar et. al. The Adaptive Threshold sensitive Energy Efficient sensor Network protocol (APTEEN) [8] aims at both capturing periodic data collections and reacting to time-critical events. The architecture is same as in TEEN. The main drawbacks of TEEN and APTEEN are the overhead and complexity of forming clusters in multiple levels implementing thresholdbased functions and dealing with attribute-based naming of queries.

Heinzelman, et. al. [9] proposed LEACHcentralized (LEACH-C), a protocol that uses a centralized clustering algorithm and the same steadystate protocol as LEACH. SEP (Stable Election Protocol) [5] is proposed in which every sensor node in a heterogeneous two-level hierarchical network independently elects itself as a cluster head based on its initial energy relative to that of other nodes. Li Qing et. al. proposed DEEC [6] (Distributed energy efficient Clustering) algorithm in which cluster head is selected on the basis of probability of ratio of residual energy and average energy of the network. Simulations show that its performance is better than other protocols. B. Elbhiri et al, proposed SBDEEC (Stochastic and Balanced Developed Distributed Energy-Efficient Clustering (SBDEEC) [10] SBDEEC introduces a balanced and dynamic method where the cluster head election probability is more efficient. Moreover, it uses a stochastic scheme detection to extend the network lifetime. Simulation results show that this protocol performs better than the Stable Election Protocol (SEP) and the Distributed Energy- Efficient Clustering (DEEC) in terms of network lifetime.

Our E-DEEC (Enhanced Distributed Energy Efficient Clustering) scheme is based on DEEC with addition of super nodes. We have extended the DEEC to three-level heterogeneity. Simulation results show that E-DEEC performs better than SEP which is too extended to three-level scheme.

\section{Radio Energy Dissipation Model}

Radio Energy Model used is based on [2, 9]. Energy model for the radio hardware energy dissipation where the transmitter dissipates energy to run the radio electronics and the power amplifier, and the receiver dissipates energy to run the radio electronics is shown in Figure 1 [2, 9].

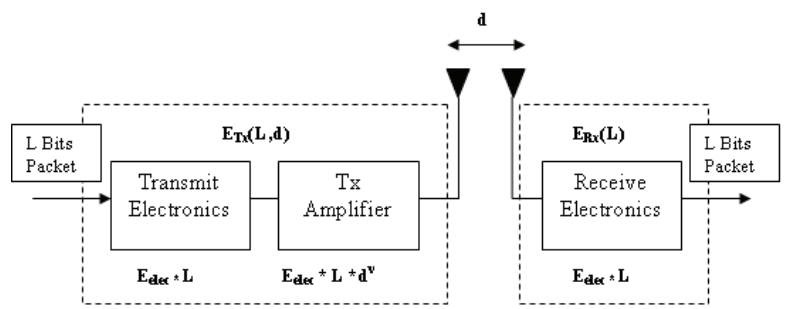

Figure 1. Radio Energy Dissipation Model

Here both the free space $\left(\mathrm{d}^{2}\right.$ power loss) and the multipath fading ( $\mathrm{d}^{4}$ power loss) channel models were used, depending on the distance between the transmitter and receiver [2, 9]. Power control can be used to invert this loss by appropriately setting the power amplifier-if the distance is less than a threshold $\mathrm{d}_{0}$, the free space model is used; otherwise, the multipath model is used. Thus, to transmit an L-bit message a distance, the radio expends

$$
E_{T x}(L, d)= \begin{cases}L \cdot E_{e l e c}+L \cdot E_{f s} \cdot d^{2} & \text { if } d<d o \\ L \cdot E_{\text {elec }}+L \cdot E_{a m p} \cdot d^{4} & \text { if } d \geq d o\end{cases}
$$

The electronics energy, $\mathrm{E}_{\mathrm{elec}}$, depends on factors such as the digital coding, modulation, filtering, and spreading of the signal, whereas the amplifier energy, $\mathrm{E}_{\mathrm{fs}} \cdot \mathrm{d}^{2}$ or $\mathrm{E}_{\mathrm{amp}} \cdot \mathrm{d}^{4}$, depends on the distance to the receiver and the acceptable bit-error rate $[2,9]$.

Value of threshold distance $d_{o}$ is given by

$$
\mathrm{do}=\frac{\mathrm{E}_{\mathrm{fs}}}{\mathrm{E}_{\mathrm{amp}}}
$$

\section{Network Model}

Sensor network is used with $\mathrm{N}$ nodes in $\mathrm{M} \mathrm{X} \mathrm{M}$ network field as shown in Figure 2.

There are three types of sensor nodes [11, 12]. They are normal nodes, advanced nodes and super nodes. Let $\mathrm{m}$ be the fraction of the total number of nodes $\mathrm{N}$, and mo is the percentage of the total number of nodes which are equipped with $b$ times more energy than the normal nodes, called as super nodes, the number is N.m.mo. The rest N.m. $(1-\mathrm{mo})$ nodes are equipped with a times more energy than the normal 
nodes; called as advanced nodes and remaining N.(1m) as normal nodes.

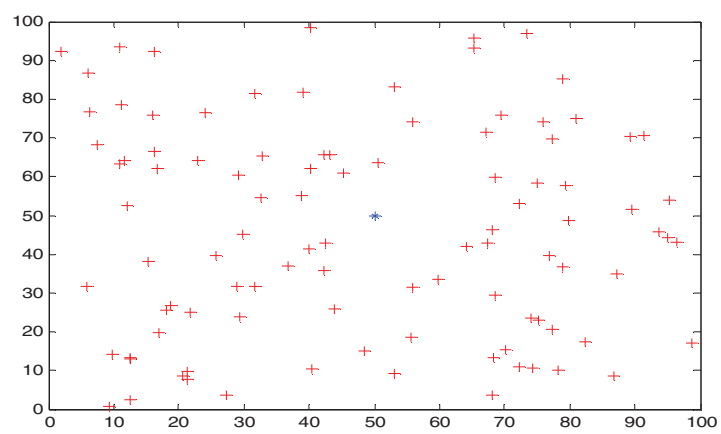

Figure 2. Random Wireless Sensor network

The total initial energy of the three-level heterogeneous networks is given by:

$$
\begin{aligned}
\mathrm{E}_{\text {total }}= & \mathrm{N} \cdot(1-\mathrm{m}) \cdot \mathrm{E}_{\mathrm{o}}+\mathrm{N} \cdot \mathrm{m} \cdot(1-\mathrm{mo}) \cdot(1+\mathrm{a}) \cdot \mathrm{E}_{\mathrm{o}} \\
& +\mathrm{N} \cdot \mathrm{m} \cdot \mathrm{mo} \cdot \mathrm{E}_{\mathrm{o}} \cdot(1+\mathrm{b}) \\
= & \mathrm{N} \cdot \mathrm{E}_{\mathrm{o}} \cdot(1+\mathrm{m} \cdot(\mathrm{a}+\mathrm{mo} \cdot \mathrm{b}))
\end{aligned}
$$

Therefore, the three-level heterogeneous networks have $\mathrm{m}(\mathrm{a}+\mathrm{mob})$ times more energy or we can say that the total energy of the system is increased by a factor of $(1+\mathrm{m} .(\mathrm{a}+\mathrm{mo} . \mathrm{b}))[11,12]$.

\section{Assumptions and Properties of the Network}

In the network model described in previous section some assumptions have been made for the sensor nodes as well as for the network. Hence the assumptions and properties of the network and sensor nodes are:

- Sensor Nodes are uniformly randomly deployed in the network.

- There is one Base Station which is located at the centre of the sensing field.

- Nodes always have the data to send to the base station.

- Nodes are location-unaware, i.e. not equipped with GPS-capable antennae.

- All nodes have similar capabilities in terms of processing and communication and of equal significance. This motivates the need for extending the lifetime of every sensor.

Sensor nodes have heterogeneity in terms of energy i.e., different energy levels. All nodes have different initial energy; some nodes are equipped with more energy than the normal nodes.

\section{Cluster Head Selection Method}

Traditionally as per LEACH, Cluster head algorithm is broken into rounds. At each round node decides whether to become a cluster head based on threshold calculated by the suggested percentage of cluster heads for the network (determined a priori) and the number of times the node has been a cluster-head so far. This decision is made by the nodes by choosing the random number between 0 and 1 . If the number is less than a threshold $\mathrm{T}(\mathrm{s})$ the node becomes a clusterhead for the current round. The threshold is set as:

$$
T(s)=\left\{\begin{array}{cl}
\frac{p}{1-p \cdot\left(r \bmod \frac{1}{p}\right)} & \text { if } s \in G \\
0 & \text { Otherwise }
\end{array}\right.
$$

where $\mathrm{p}, \mathrm{r}$, and $\mathrm{G}$ represent, respectively, the desired percentage of cluster-heads, the current round number, and the set of nodes that have not been cluster-heads in the last $1 / \mathrm{p}$ rounds. Using this threshold, each node will be a cluster head, just once at some point within $1 / \mathrm{p}$ rounds.

In the three level heterogeneous networks there are three types of nodes normal nodes, advanced nodes and super nodes, as discussed in section 4, based on their initial energy. Hence the reference value of $p$ is different for these types of nodes. The probabilities of normal, advanced and super nodes are:

$p_{i}= \begin{cases}\frac{p_{\text {opt }} E_{i}(r)}{(1+m \cdot(a+m o . b)) \bar{E}(r)} & \text { if } \quad s_{i} \text { is the normal node } \\ \frac{p_{\text {opt }}(1+a) E_{i}(r)}{(1+m \cdot(a+m o . b)) \bar{E}(r)} & \text { if } \quad s_{i} \text { is the advanced node (5) } \\ \frac{p_{\text {opt }}(1+b) E_{i}(r)}{(1+m \cdot(a+m o . b)) \bar{E}(r)} & \text { if } s_{i} \text { is the super node }\end{cases}$

Threshold for cluster head selection is calculated for normal, advanced, super nodes by putting above values in Eq. (4).

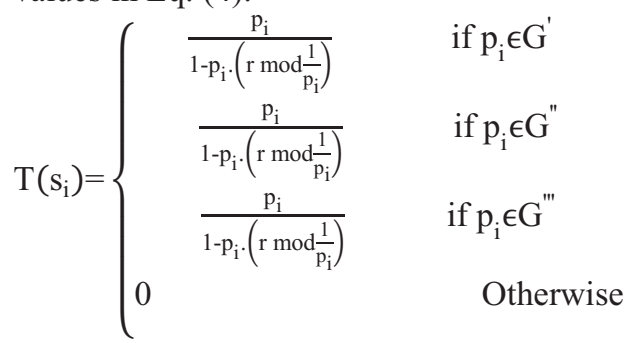

where $G^{\prime}$ is the set of normal nodes that have not become cluster heads within the last $1 / p_{i}$ rounds of the epoch where $\mathrm{s}_{\mathrm{i}}$ is normal node, $\mathrm{G}^{\prime \prime}$ is the set of advanced nodes that have not become cluster heads within the last $1 / p_{i}$ rounds of the epoch where $s_{i}$ is advanced node, $\mathrm{G}^{\prime \prime \prime}$ is the set of super nodes that have not become 
cluster heads within the last $1 / p_{i}$ rounds of the epoch where $\mathrm{s}_{\mathrm{i}}$ is super node.

E-DEEC implements the same strategy for estimating the energy in the network as proposed in DEEC [6]. Since the probabilities calculated depend on the average energy of the network at round $r$, hence this is to be calculated. This average energy is estimated as:

$$
\bar{E}(r)=\frac{1}{N} E_{\text {total }}\left(1-\frac{r}{R}\right)
$$

where $\mathrm{R}$ denotes the total rounds of the network lifetime. $\mathrm{R}$ can be calculated as

$$
\mathrm{R}=\frac{\mathrm{E}_{\text {total }}}{\mathrm{E}_{\text {round }}}
$$

$\mathrm{E}_{\text {round }}$ is the energy dissipated in the network in a round.

The total energy dissipated $\mathrm{E}_{\text {round }}$ is equal to

$$
\mathrm{E}_{\text {round }}=\mathrm{L}\left(2 \mathrm{NE}_{\text {elec }}+\mathrm{NE}_{\mathrm{DA}}+\mathrm{kE}_{\mathrm{amp}} \mathrm{d}_{\mathrm{toBS}}^{4}+\mathrm{NE}_{\mathrm{fs}} \mathrm{d}_{\mathrm{toCH}}^{2}\right)
$$

where $\mathrm{k}$ is number of clusters $\mathrm{d}_{\mathrm{toBS}}$ is the average distance between cluster head and the base station and $\mathrm{d}_{\mathrm{toCH}}$ is the average distance between the cluster members and the cluster head.

$$
\text { Now, } \mathrm{d}_{\mathrm{toCH}}=\frac{\mathrm{M}}{\sqrt{2 \pi \mathrm{k}}}, \mathrm{d}_{\mathrm{toBS}}=0.765 \frac{\mathrm{M}}{2}
$$

By calculating the derivative of $E_{\text {round }}$ with respect to $k$ to zero we get optimal number of clusters as

$$
\mathrm{k}_{\mathrm{opt}}=\sqrt{\frac{\mathrm{N}}{2 \pi}} \frac{\mathrm{M}}{\mathrm{d}_{\mathrm{toBS}}^{2}} \sqrt{\frac{\mathrm{E}_{\mathrm{fs}}}{\mathrm{E}_{\mathrm{amp}}}}
$$

Hence we can find the energy dissipated per round by substituting equations (10) \& (11) in (9).Due to the heterogeneity factors $\mathrm{R}$ is taken as $1.5 \mathrm{R}$ (Since $\overline{\mathrm{E}}(\mathrm{r})$ will be too large at the end from Eq.(7), some will not die finally)

\section{Performance Criteria Used}

The performance metrics or parameters used to study and evaluate the clustering protocols are lifetime, number of nodes alive and number of data packets received at base station.

- Data Packets received at base station: It is total number of data packets or messages that are received by the base station. This measure varies linearly for all protocols.

- Number of alive nodes: This instantaneous measure reflects the total number of nodes and that of each type that has not yet expended all of their energy.
- Network remaining energy: It measures the total remaining energy of the network. It is calculated at each transmission round of the protocol.

These metrics used allow us to conclude about the stability period of the network which is the time interval from the start of network operation until the death of the first sensor node, unstable period of the network which is the time interval from the death of the first node until the death of the last node, energy consumption, the data send that are received by the base station [5] and the lifetime of the network which is number of rounds until the first node die which is simply the stability period of the network ( We have assume all the nodes having equal importance). More stable is the network; more is the lifetime of the network.

Table 1. Simulation Parameters

\begin{tabular}{|l|l|}
\hline Parameters & Value \\
\hline Network Field & $(100,100)$ \\
\hline Number of nodes & 100 \\
\hline $\begin{array}{l}\text { Eo ( Initial energy of } \\
\text { normal nodes) }\end{array}$ & $0.5 \mathrm{~J}$ \\
\hline Message Size & $4000 \mathrm{Bits}$ \\
\hline $\mathrm{E}_{\text {elec }}$ & $50 \mathrm{~nJ} / \mathrm{bit}$ \\
\hline $\mathrm{E}_{\mathrm{fs}}$ & $10 \mathrm{~nJ} / \mathrm{bit} / \mathrm{m}^{2}$ \\
\hline $\mathrm{E}_{\mathrm{amp}}$ & $0.0013 \mathrm{pJ} / \mathrm{bit} / \mathrm{m}^{4}$ \\
\hline $\mathrm{E}_{\mathrm{DA}}$ & $5 \mathrm{~nJ} / \mathrm{bit} / \mathrm{signal}$ \\
\hline $\mathrm{d}_{\mathrm{o}}($ Threshold Distance) $)$ & $70 \mathrm{~m}$ \\
\hline $\mathrm{p}_{\text {opt }}$ & 0.1 \\
\hline
\end{tabular}

\section{Simulation and Results}

We have simulated our wireless sensor network in a sensing field of $100 \mathrm{~m} \times 100 \mathrm{~m}$. Simulation parameters used are listed in table 1 . In our scenario, we have deployed $20 \%$ advanced nodes deployed with 1.5 times more energy than normal nodes and 30\% super nodes deployed with 3 times more energy than the normal nodes $(\mathrm{m}=0.5, \mathrm{mo}=0.4, \mathrm{a}=1.5, \mathrm{~b}=3)$. Hence more total initial energy. We have compared E-DEEC with SEP which is too extended to three-level based on the same approach.

Figure 3 represent the number of nodes alive during the lifetime of the network. It clearly shows that by introducing super nodes lifetime increases. Stability period and lifetime of EDEEC is longer as compared to SEP and unstable period of SEP is longer than EDEEC. EDEEC is better than SEP as it uses the residual energy. In SEP death of nodes starts after 1200 rounds while for EDEEC it starts after 1500 rounds. Last node for SEP and E-DEEC dies at 6000 and 4100 rounds. 


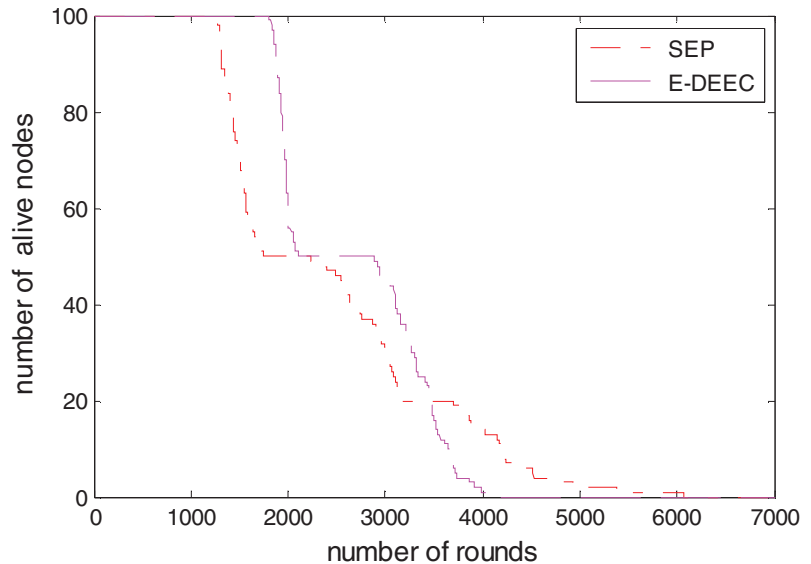

Figure 3. Number of nodes alive over rounds under three-level heterogeneity of SEP and E-DEEC

Figure 4 shows the comparison in terms of number of data packets received at the base station. The results show that for both the protocols it goes linearly for around 3000 rounds and after that the difference can be seen. It is clear E-DEEC has more numbers of data packets received at base station in comparison to SEP.

Figure 5 show total remaining energy over time i.e., number of rounds. Here total initial energy is $102.5 \mathrm{~J}$ which decreases linearly up to around 2000 rounds for both E-DEEC and SEP. Energy per round is more in EDEEC as compared to SEP up to around 3000 rounds then graph changes for both E-DEEC and SEP from the round where first node dies in respect to them. Most of the energy is consumed in the first 3000 rounds.

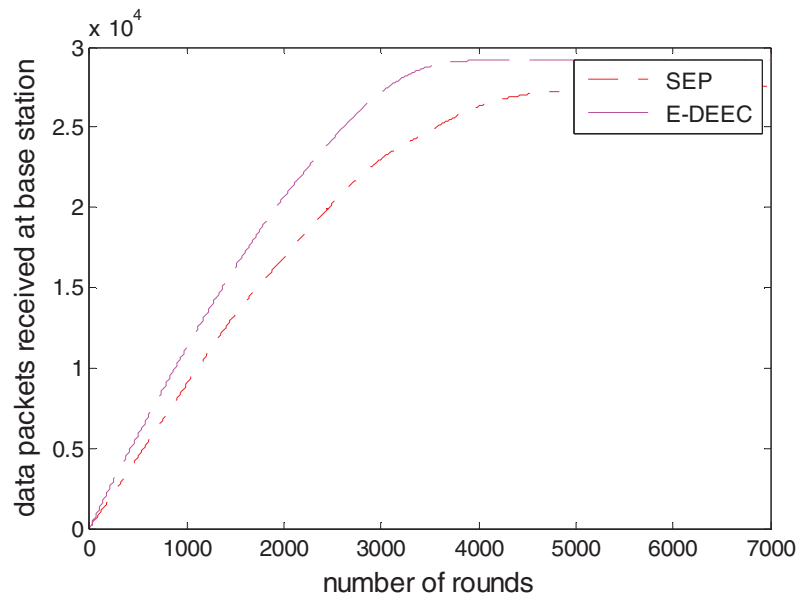

Figure 4. Data Packets over rounds under three-level heterogeneity of SEP and E-DEEC

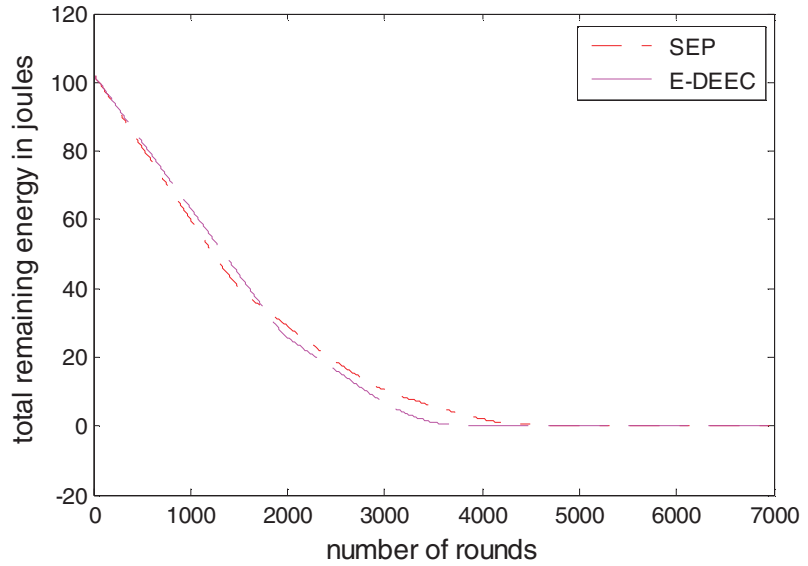

Figure 5. Total remaining energy over rounds under three-level heterogeneity of SEP and E-DEEC

\section{Conclusion}

Wireless sensor network is a combination of wireless communication and sensor nodes. The network should be energy efficient with stability and longer lifetime. In this paper, proposed E-DEEC adds heterogeneity in the network by introducing the super nodes having energy more than normal and advanced nodes and respective probabilities. Simulation results shows that E-DEEC has better performance as compared to SEP in terms of parameters used. It extends the lifetime and stability of the network.

\section{References}

[1] Jamal N. Al-Karaki, Ahmed E. Kamal," Routing Techniques In Wireless Sensor Networks: A Survey", IEEE Wireless Communications, Volume: 11, Issue: 6, 26- 28, December 2004.

[2] W.Heinzelman, A. Chandrakasan, and H. Balakrishnan, "Energy-efficient communication protocol for wireless sensor networks," in the Proceeding of the Hawaii International Conference System Sciences, Hawaii, January 2000 .

[3] S. Lindsey and C. S. Raghavendra, "PEGASIS: Power Efficient Gathering in Sensor Information Systems," in the Proceedings of the IEEE Aerospace Conference, Big Sky, Montana, March 2002.

[4] O. Younis, S. Fahmy, "HEED: A hybrid, energy efficient, distributed clustering approach for adhoc sensor networks", IEEE Transactions on Mobile Computing vol 3, no 4, pp 660-669, 2004.

[5] G. Smaragdakis, I. Matta, A. Bestavros, "SEP: A Stable Election Protocol for clustered heterogeneous wireless sensor networks", in: Second International Workshop on Sensor and 
Actor Network Protocols and Applications (SANPA 2004), 2004.

[6] L. Qing, Q. Zhu, M. Wang, "Design of a distributed energy-efficient clustering algorithm for heterogeneous wireless sensor networks". ELSEVIER, Computer Communications 29, 2006, pp 2230- 2237.

[7] A. Manjeshwar and D. P. Agarwal, "TEEN: a routing protocol for enhanced efficiency in wireless sensor networks," In 1st International Workshop on Parallel and Distributed Computing Issues in Wireless Networks and Mobile Computing, April 2001.

[8] A. Manjeshwar and D. P. Agarwal, "APTEEN: A hybrid protocol for efficient routing and comprehensive information retrieval in wireless sensor networks," Parallel and Distributed Processing Symposium., Proceedings International, IPDPS 2002, pp. 195-202.

[9] W.R. Heinzelman, A.P. Chandrakasan, H. Balakrishnan, An application specific protocol architecture for wireless microsensor networks, IEEE Transactions on Wireless Communications 1 (4) (2002) 660-670.
[10] Elbhiri Brahim,Saadane Rachid,Alba-Pages Zamora, Driss Aboutajdine, "Stochastic Distributed Energy-Efficient Clustering (SDEEC) for heterogeneous wireless sensor networks", ICGST-CNIR Journal, Volume 9, Issue 2, December 2009.

[11] Yingchi Mao, Zhen Liu, Lili Zhang, Xiaofang Li, “An Effective Data Gathering Scheme in Heterogeneous Energy Wireless Sensor Networks, International Conference on Computational Science and Engineering,2009.

[12] Dilip Kumar, Trilok C. Aseri, R.B. Patel, "EEHC: Energy efficient heterogeneous clustered scheme for wireless sensor networks", ELSEVIER, Computer Communications, 32 (2009) 662-667. 